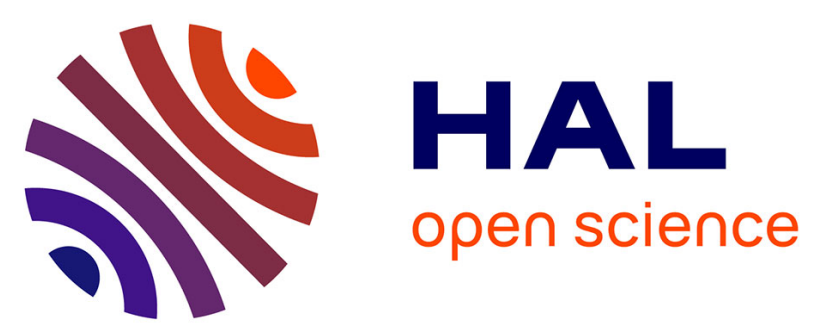

\title{
Reduced-order computational model in nonlinear structural dynamics for structures having numerous local elastic modes in the low-frequency range. Application to fuel assemblies
}

Anas Batou, Christian Soize, N. Brie

\section{To cite this version:}

Anas Batou, Christian Soize, N. Brie. Reduced-order computational model in nonlinear structural dynamics for structures having numerous local elastic modes in the low-frequency range. Application to fuel assemblies. Nuclear Engineering and Design, 2013, 262 (-), pp.276-284. 10.1016/j.nucengdes.2013.04.039 . hal-00821274

\section{HAL Id: hal-00821274 \\ https://hal.science/hal-00821274}

Submitted on 8 May 2013

HAL is a multi-disciplinary open access archive for the deposit and dissemination of scientific research documents, whether they are published or not. The documents may come from teaching and research institutions in France or abroad, or from public or private research centers.
L'archive ouverte pluridisciplinaire HAL, est destinée au dépôt et à la diffusion de documents scientifiques de niveau recherche, publiés ou non, émanant des établissements d'enseignement et de recherche français ou étrangers, des laboratoires publics ou privés. 


\title{
Reduced-order computational model in nonlinear structural dynamics for structures having numerous local elastic modes in the low-frequency range. Application to fuel assemblies
}

\author{
A. Batou*a, C. Soize ${ }^{\mathrm{a}}$, N. Brie ${ }^{\mathrm{b}}$ \\ ${ }^{a}$ Université Paris-Est, Laboratoire Modélisation et Simulation Multi Echelle, MSME \\ UMR 8208 CNRS, 5 bd Descartes, 77454 Marne-la-Vallee, France. \\ ${ }^{b}$ EDF RED, Département AMA, 1 avenue du général De Gaulle, 92140 Clamart, France.
}

\begin{abstract}
We are interested in the construction of a reduced-order computational model for nonlinear complex dynamical structures which are characterized by the presence of numerous local elastic modes in the low-frequency band. This high modal density makes the use of the classical modal analysis method not suitable. Therefore the reduced-order computational model is constructed using a basis of a space of global displacements, which is constructed a priori and which allows the nonlinear dynamical response of the structure observed on the stiff part to be predicted with a good accuracy. The methodology is applied to a complex industrial structure which is made up of a row of seven fuel assemblies with possibility of collisions between grids and which is submitted to a seismic loading.
\end{abstract}

Keywords: reduced-order model, nonlinear structural dynamics, global elastic modes, local elastic modes, fuel assemblies

\section{Introduction}

This paper is devoted to the construction of a reduced-order computational model for low-frequency nonlinear structural dynamics in presence of

\footnotetext{
*Corresponding author

Email addresses: anas.batou@univ-paris-est.fr (A. Batou*), christian.soize@univ-paris-est.fr (C. Soize), nicolas.brie@edf.fr (N. Brie) 
numerous local elastic modes. We are interested in structures which are made up of a rigid frame (the stiff part) coupled with several flexible substructures. The dynamical behavior of these kind of structures is characterized by a high modal density in the low-frequency range. More precisely, these structures exhibit some global elastic modes and numerous local elastic modes in the same low-frequency range. Moreover, the structure we consider is modeled with a large finite element model and has several localized nonlinearities (such as elastic stops). As a consequence, the direct construction of the transient response is a very challenging issue since the presence of the localized nonlinearities requires a small time step for the integration scheme in order to correctly capture the nonlinear effects. We then have to construct a reduced-order computational model for a large computational model. For such a structure, the reduction of the nonlinear dynamical computational model can be carried out using the elastic modes of the underlying linear part of the nonlinear dynamical system.

The objective of this paper is to construct a reduced-order computational model with a very small dimension and which has the capability to predict the nonlinear dynamical responses of the structure observed on the stiff part with a good accuracy. Since the contributions of the local displacements are negligible in the displacements of the stiff part, we have to construct the reduced-order computational model using a basis adapted to the prediction of the global displacements and therefore, we have to filter the local displacements in the construction of the basis. To achieve this objective, most of previous researches have been based on a spatial filtering of the short wavelengths. Concerning the experimental methods, such a filtering is carried out using regularization techniques [5], image-based finite element methods [11] or an extraction of eigenvectors of the frequency mobility matrix [10]. Concerning numerical methods, most of the techniques are based on the lumped mass methods. In the Guyan method [8], the masses are lumped at a few nodes and the inertia forces of the other nodes are neglected. It should be noted that the choice of points in which the masses are concentrated is not obvious to do for complex structures $[4,22,18]$. The convergence properties of the solution obtained using the lumped mass method have been studied $[6,13,3]$. In [17], the authors propose to construct a basis of the global displacements space using a rough finite element model. For slender dynamical structures, another method consists in the construction of an equivalent beam or plate model $[21,23]$. In [9], the author circumvent the problem of the high modal density by extrapolating the dynamical response using a few 
elastic modes. This method is interesting when one has an analytical expression for the modal shape, that is not the case for the structures considered in this paper. In [14], the authors use a free-interface substructuring method in order to extract long wavelength free modes of a master structure. The Proper Orthogonal Decomposition (POD) method (see [15, 19, 12]) allows in some cases to extract an accurate small size basis in order to construct a reduced-order computational model of a nonlinear dynamical system (see for instance $[1,16,20,24])$, but this basis has to be constructed a posteriori, which means that a sufficiently rich nonlinear response has to be constructed. Moreover, the POD basis is only optimal for a given external load (or imposed displacement). In [25], a similar method to POD is used for linear dynamical systems but the basis which is constructed is independent of any given external load.

Recently, a new method has been proposed to construct a reduced-order computational model in linear structural dynamics for structures having numerous local elastic modes in the low-frequency band [26]. In this method, a basis of the space of global displacements and a basis of the space of local displacements are calculated by solving two separated eigenvalues problems. The elements of these two bases are not constituted of the usual elastic modes. The eigenvalue problem, allowing a basis of the space of global displacements to be defined, is constructed by introducing a kinematic reduction for calculating the kinetic energy while the elastic energy is kept exact. In this paper, this method will be used to construct a basis of the space of global displacements and then to deduce the reduced-order computational model of the nonlinear dynamical system under consideration. Therefore, the contributions of the local displacements of the structure observed on the stiff part will be neglected.

In Section 2, the method developed in [26] is summarized and the construction of the reduced-order computational model is presented. In Section 3 , the methodology is developed for an industrial structure for which the nonlinear transient response of a row of fuel assemblies is analyzed.

\section{Construction of the reduced-order computational model}

In this Section, we first summarize the method introduced in [26] which allows a reduced-order computational model to be constructed for structures having a high modal density in the low-frequency range. This method has been developed for the continuous case and for the discrete case. Below, we 
just summarize the discrete case. Moreover, although this method allows both a basis of the space of global displacements and a basis of the space of local displacements to be constructed (by solving two separated eigenvalue problems), we will use only the basis of the space of global displacements to construct the reduced-order model.

\subsection{Reference nonlinear computational model}

We are interested in predicting the transient responses of a three-dimensional nonlinear damped structure, with localized nonlinearities, and occupying a bounded domain $\Omega$. The real-valued vector $\mathbb{U}(t)$ of the $m$ degrees of freedom (DOFs) of the computational model is constructed with the finite element method and is the solution of the following matrix equation,

$$
\left.\left.[\mathbb{M}] \ddot{\mathbb{U}}(t)+[\mathbb{D}] \dot{U}(t)+[\mathbb{K}] \mathbb{U}(t)+\mathbb{F}^{\mathrm{NL}}(\mathbb{U}(t), \dot{U}(t))=\mathbb{F}(t), t \in\right] 0, T\right],
$$

with the initial conditions

$$
\mathbb{U}(0)=\dot{U}(0)=\mathbf{0},
$$

in which $[\mathbb{M}]$, $[\mathbb{D}]$ and $[\mathbb{K}]$ are respectively the $(m \times m)$ positive-definite symmetric real mass, damping and stiffness matrices, where $\mathbb{F}^{\mathrm{NL}}(\mathbb{U}(t), \dot{U}(t))$ is the vector of the nonlinear forces induced by the localized nonlinearities and where $\mathbb{F}(t)$ is relative to the discretization of the external forces. Usually, the nonlinear matrix equation (1) is reduced using the elastic modes of the linear part of Eq. (1). These modes are therefore the solutions of the generalized eigenvalue problem, $([\mathbb{K}]-\lambda[\mathbb{M}]) \boldsymbol{\varphi}=\mathbf{0}$. As it is assumed that there are numerous local elastic modes and as there are nonlinear forces, one would need to calculate a high number of elastic modes in order to obtain a good convergence for the nonlinear dynamical response in the low-frequency range. The use of a basis of the space of global displacements circumvents this difficulty.

\subsection{Kinematic reduction of the kinetic energy}

The methodology proposed in [26] consists in introducing a kinematic reduction of the structural kinetic energy. In a first step, the domain $\Omega$ is partitioned into $n_{J}$ disjoint subdomains $\Omega_{j}$. In a second step, this decomposition is used to construct the projection linear operator, $\boldsymbol{u} \mapsto h^{r}(\boldsymbol{u})$, such 
that $h^{r}\left(h^{r}(\boldsymbol{u})\right)=h^{r}(\boldsymbol{u})$ and defined by

$$
\left\{h^{r}(\boldsymbol{u})\right\}(\boldsymbol{x})=\sum_{j=1}^{n_{J}} \mathbb{1}_{\Omega_{j}}(\boldsymbol{x}) \frac{1}{m_{j}} \int_{\Omega_{j}} \rho\left(\boldsymbol{x}^{\prime}\right) \boldsymbol{u}\left(\boldsymbol{x}^{\prime}\right) d \boldsymbol{x}^{\prime},
$$

in which $\boldsymbol{x} \mapsto \mathbb{1}_{\Omega_{j}}(\boldsymbol{x})=1$ if $\boldsymbol{x}$ is in $\Omega_{j}$ and $=0$ otherwise, where $m_{j}$ is the total mass of subdomain $\Omega_{j}$ and where $\rho(\boldsymbol{x})$ is the mass density. This operator carries out an average of the displacements with respect to the mass density in each subdomain (kinematic reduction). Then, the complementary projection linear operator, $\boldsymbol{u} \mapsto h^{c}(\boldsymbol{u})$, is defined by

$$
h^{c}(\boldsymbol{u})=\boldsymbol{u}-h^{r}(\boldsymbol{u}) .
$$

Function $h^{r}(\boldsymbol{u})$ will also be denoted by $\boldsymbol{u}^{r}$ and function $h^{c}(\boldsymbol{u})$ by $\boldsymbol{u}^{c}$. We then have $\boldsymbol{u}=h^{r}(\boldsymbol{u})+h^{c}(\boldsymbol{u})$ that is to say, $\boldsymbol{u}=\boldsymbol{u}^{r}+\boldsymbol{u}^{c}$ with $\boldsymbol{u}^{r}=h^{r}(\boldsymbol{u})$ and $\boldsymbol{u}^{c}=h^{c}(\boldsymbol{u})$. Let $\left[H^{r}\right]$ be the $(m \times m)$ matrix corresponding to the finite element discretization of the projection operator $h^{r}$ defined by Eq. (3). Therefore, the vector $\mathbb{U}$ can be written as $\mathbb{U}=\mathbb{U}^{r}+\mathbb{U}^{c}$, in which

$$
\mathbb{U}^{r}=\left[H^{r}\right] \mathbb{U}
$$

and

$$
\mathbb{U}^{c}=\left[H^{c}\right] \mathbb{U}=\mathbb{U}-\mathbb{U}^{r},
$$

which shows that $\left[H^{c}\right]=\left[I_{m}\right]-\left[H^{r}\right]$, where $\left[I_{m}\right]$ is the $(m \times m)$ identity matrix. Then, the $(m \times m)$ mass matrix $\left[\mathbb{M}^{r}\right]$ is such that

$$
\left[\mathbb{M}^{r}\right]=\left[H^{r}\right]^{T}[\mathbb{M}]\left[H^{r}\right]
$$

and the complementary $(m \times m)$ mass matrix $\left[\mathbb{M}^{c}\right]$ is such that

$$
\left[\mathbb{M}^{c}\right]=\left[H^{c}\right]^{T}[\mathbb{M}]\left[H^{c}\right] .
$$

Using the properties of the projection operator defined by Eq. (3), it can be shown [26] that

$$
\begin{gathered}
{\left[\mathbb{M}^{c}\right]=[\mathbb{M}]-\left[\mathbb{M}^{r}\right]} \\
{\left[\mathbb{M}^{r}\right]=[\mathbb{M}]\left[H^{r}\right]=\left[H^{r}\right]^{T}[\mathbb{M}] .}
\end{gathered}
$$


The rank of the matrix $\left[\mathbb{M}^{r}\right]$ is $3 n_{J}$ and the rank of the matrix $\left[\mathbb{M}^{c}\right]$ is $m-3 n_{J}$. Some details concerning the construction of matrices $\left[H^{r}\right],\left[H^{c}\right]$, $\left[\mathbb{M}^{r}\right]$ and $\left[\mathbb{M}^{c}\right]$ are given in Appendix $\mathrm{A}$.

\subsection{Basis of the space of global displacements}

The basis of the space of global displacements is made up of the solutions $\varphi^{g}$ in $\mathbb{R}^{m}$ of the generalized eigenvalue problem

$$
[\mathbb{K}] \varphi^{g}=\lambda^{g}\left[\mathbb{M}^{r}\right] \varphi^{g},
$$

in which the stiffness matrix is kept exact while the mass matrix is projected. This generalized eigenvalue problem admits an increasing sequence of $3 n_{J}$ positive global eigenvalues $0<\lambda_{1}^{g} \leq \ldots \leq \lambda_{3 n_{J}}^{g}$, associated with the finite family of algebraically independent eigenvectors $\left\{\varphi_{1}^{g}, \ldots, \varphi_{3 n_{J}}^{g}\right\}$. The family $\left\{\boldsymbol{\varphi}_{1}^{g}, \ldots, \boldsymbol{\varphi}_{3 n_{J}}^{g}\right\}$ spans a subspace of dimension $3 n_{J}$, which is defined as the space of global displacements. In general, this family is not made up of the elastic modes. The computation of the eigenvectors is carried out using an adapted subspace iteration algorithm which is detailed in Appendix B. This algorithm avoid the assembly of matrix $\left[\mathbb{M}^{r}\right]$ which is a full matrix. If needed, a basis of the space of local displacements can also be constructed and complements the basis of global displacements (see [26]). In this case, the basis of the space of local displacements is made up of the solutions $\phi^{\ell}$ in $\mathbb{R}^{m}$ of the generalized eigenvalue problem

$$
[\mathbb{K}] \varphi^{\ell}=\lambda^{\ell}\left[\mathbb{M}^{c}\right] \varphi^{\ell}
$$

This generalized eigenvalue problem admits an increasing sequence of positive eigenvalues $0<\lambda_{1}^{\ell} \leq \ldots \leq \lambda_{m-3 n_{J}}^{\ell}$, associated with the algebraically independent eigenvectors $\left\{\boldsymbol{\varphi}_{1}^{\ell}, \ldots, \boldsymbol{\varphi}_{m-3 n_{J}}^{\ell}\right\}$. The family $\left\{\boldsymbol{\varphi}_{1}^{\ell}, \ldots, \boldsymbol{\varphi}_{m-3 n_{J}}^{\ell}\right\}$ spans a subspace of dimension $m-3 n_{J}$ defined as the space of local displacements. In general, this family is not made up of the elastic modes.

\subsection{Reduced-order computational model}

In [26], it is proven that the family $\left\{\boldsymbol{\varphi}_{1}^{g}, \ldots, \boldsymbol{\varphi}_{3 n_{J}}^{g}, \boldsymbol{\varphi}_{1}^{\ell}, \ldots, \boldsymbol{\varphi}_{m-3 n_{J}}^{\ell}\right\}$ is a basis of $\mathbb{R}^{m}$. Therefore, we can construct a reduced-order model of dimension $\left(n_{g}, n_{\ell}\right)$ which is defined as the projection $\mathbb{U}_{n_{g}, n_{\ell}}(t)$ of $\mathbb{U}(t)$ on the subspace of $\mathbb{R}^{m}$ spanned by the family $\left\{\boldsymbol{\varphi}_{1}^{g}, \ldots, \boldsymbol{\varphi}_{n_{g}}^{g}, \boldsymbol{\varphi}_{1}^{\ell}, \ldots, \boldsymbol{\varphi}_{n_{\ell}}^{\ell}\right\}$ of real vectors associated with the first $n_{g}$ global eigenvectors such that $n_{g} \leq 3 n_{J} \leq m$ and 
with the first $n_{\ell}$ local eigenvectors such that $n_{\ell} \leq m-3 n_{J}$. Then, the approximation $\mathbb{U}_{n_{g}, n_{\ell}}(t)$ of $\mathbb{U}(t)$ at order $\left(n_{g}, n_{\ell}\right)$ is written as

$$
\mathbb{U}_{n_{g}, n_{\ell}}(t)=\sum_{\alpha=1}^{n_{g}} q_{\alpha}^{g}(t) \varphi_{\alpha}^{g}+\sum_{\beta=1}^{n_{\ell}} q_{\beta}^{\ell}(t) \boldsymbol{\varphi}_{\beta}^{\ell},
$$

where $q_{1}^{g}(t), \ldots, q_{n_{g}}^{g}(t)$ are the global generalized coordinates and where $q_{1}^{\ell}(t)$, $\ldots, q_{n_{\ell}}^{\ell}(t)$ are the local generalized coordinates. In this paper, we are only interested in the prediction of the global behavior of a nonlinear dynamical structure, that is to say in the nonlinear dynamical responses of the stiff part of the structure and consequently, the local contributions are neglected which means that $n_{\ell}=0$. Let $\left[\Phi^{g}\right]=\left[\boldsymbol{\varphi}_{1}^{g} \ldots \varphi_{n_{q}}^{g}\right]$ be the $\left(m \times n_{g}\right)$ real matrix whose columns are the vectors $\varphi_{1}^{g}, \ldots, \varphi_{n_{g}}^{g}$. Then, the $n_{g}$-order approximation $\mathbb{U}_{n_{g}}(t)$ of $\mathbb{U}(t)$ is written as

$$
\mathbb{U}_{n_{g}}(t)=\sum_{\alpha=1}^{n_{g}} q_{\alpha}^{g}(t) \boldsymbol{\varphi}_{\alpha}^{g}=\left[\Phi^{g}\right] \boldsymbol{q}^{g}(t),
$$

in which the vector $\boldsymbol{q}^{g}(t)=\left(q_{1}^{g}(t), \ldots, q_{n_{g}}^{g}(t)\right)$ is the solution of the following nonlinear reduced matrix equation

$$
\left.\left.[M] \ddot{\boldsymbol{q}}^{g}(t)+[D] \dot{\boldsymbol{q}}^{g}(t)+[K] \boldsymbol{q}^{g}(t)+\boldsymbol{f}^{\mathrm{NL}}\left(\boldsymbol{q}^{g}(t), \dot{\boldsymbol{q}}^{g}(t)\right)=\boldsymbol{f}(t), t \in\right] 0, T\right],
$$

with the initial conditions

$$
\boldsymbol{q}^{g}(0)=\dot{\boldsymbol{q}}^{g}(0)=\mathbf{0}
$$

In Eq. (15), the generalized mass, damping and stiffness $\left(n_{g} \times n_{g}\right)$ matrices are defined by

$$
[M]=\left[\Phi^{g}\right]^{T}[\mathbb{M}][\Phi]^{g} \quad, \quad[D]=\left[\Phi^{g}\right]^{T}[\mathbb{D}][\Phi]^{g} \quad, \quad[K]=\left[\Phi^{g}\right]^{T}[\mathbb{K}][\Phi]^{g},
$$

the vector of the generalized forces is defined by $\boldsymbol{f}(t)=\left[\Phi^{g}\right]^{T} \mathbb{F}(t)$ and $\boldsymbol{f}^{\mathrm{NL}}\left(\boldsymbol{q}^{g}(t), \dot{\boldsymbol{q}}^{g}(t)\right)=\left[\Phi^{g}\right]^{T} \mathbb{F}^{\mathrm{NL}}\left([\Phi]^{g} \boldsymbol{q}^{g}(t),[\Phi]^{g} \dot{\boldsymbol{q}}^{g}(t)\right)$ is the vector of the generalized nonlinear forces. In this paper, we are interested in a nonlinear dynamical system for which the size of the nonlinear reduced-order computational model, defined by Eqs. (14) to (16), is very small. It should be noted that, as the local contributions are neglected, an irreducible error is 
introduced. As explained above, as we are only interested in the dynamical behavior of the stiff part of the structure for which the responses are mainly due to the global displacements, this error stays very small for the quantities of interest. It should also be noted that the global eigenvectors (the eigenvectors in the space of global displacements) have been constructed using the linear part of Eq. (1). Therefore, a convergence analysis of the nonlinear response must be carried out in order to verify that enough global eigenvectors have been taken into account in the construction of the reduced-order model, even if the energy of the imposed forces or imposed displacements is concentrated in a very low-frequency range.

\section{Application}

This section is devoted to an industrial application which consists in the dynamical analysis of a row of seven fuel assemblies with possible collisions between the grids. The mechanical system is submitted to a seismic loading. For reasons of confidentiality, some technical information have been intentionally omitted.

\subsection{Reference computational model}

(i)-Fuel assembly

A fuel assembly is a slender structure which is made up of 264 flexible fuel rods, 25 stiff guide tubes and 10 stiff grids which hold the tubes in position (see the finite element mesh in Fig. 1). The stiff guide tubes are welded to the grids while the flexible fuel rods are fixed to the grids by springs. The longitudinal (vertical) direction is denoted by $\boldsymbol{z}$. The transverse directions are denoted by $\boldsymbol{x}$ and $\boldsymbol{y}$. The flexible fuel rods and the stiff guide tubes are modeled by Timoshenko beams and the grids are modeled by solid elements. The end of the stiff guide tubes are fixed to the containment building. All the displacements following $\boldsymbol{y}$-direction are set to zero. For a single fuel assembly, the finite element model has 44,844 elements and 449,580 DOFs. There are 7,364 elastic modes in the frequency band $[0,400] \mathrm{Hz}$. The first eight elastic modes are elastic ensemble modes (all the structure moves in phase), the corresponding eigenfrequencies are $3.09 \mathrm{~Hz}, 6.31 \mathrm{~Hz}, 9.78 \mathrm{~Hz}, 13.5 \mathrm{~Hz}$, 17.6 Hz, 22.2 Hz, 27.3 Hz and $32.7 \mathrm{~Hz}$. Beyond these elastic ensemble modes, there are numerous local elastic modes (only a part of the structure moves) and a few global elastic modes (all the structure moves but not in phase). The cumulative modal distribution of elastic modes is plotted in Fig. 2. The 


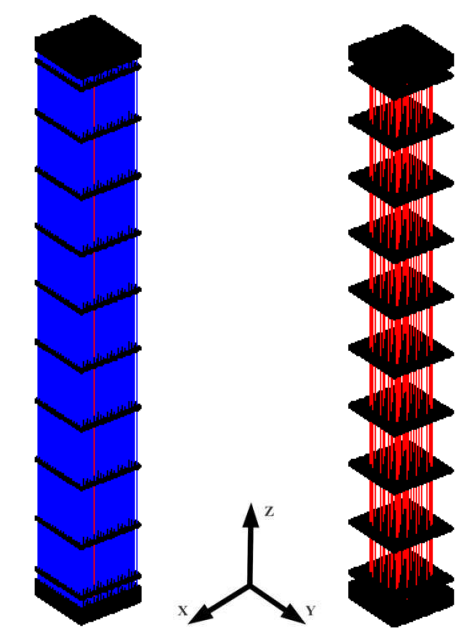

Figure 1: Finite element mesh of a fuel assembly: Grids (black), fuel rods (blue) and guide tubes (red). Left figure: Complete fuel assembly. Right figure: Grids and guide tubes only.

$2^{\text {nd }}$ elastic mode (global) and the $20^{\text {th }}$ elastic mode (local) are plotted in Fig. 3.

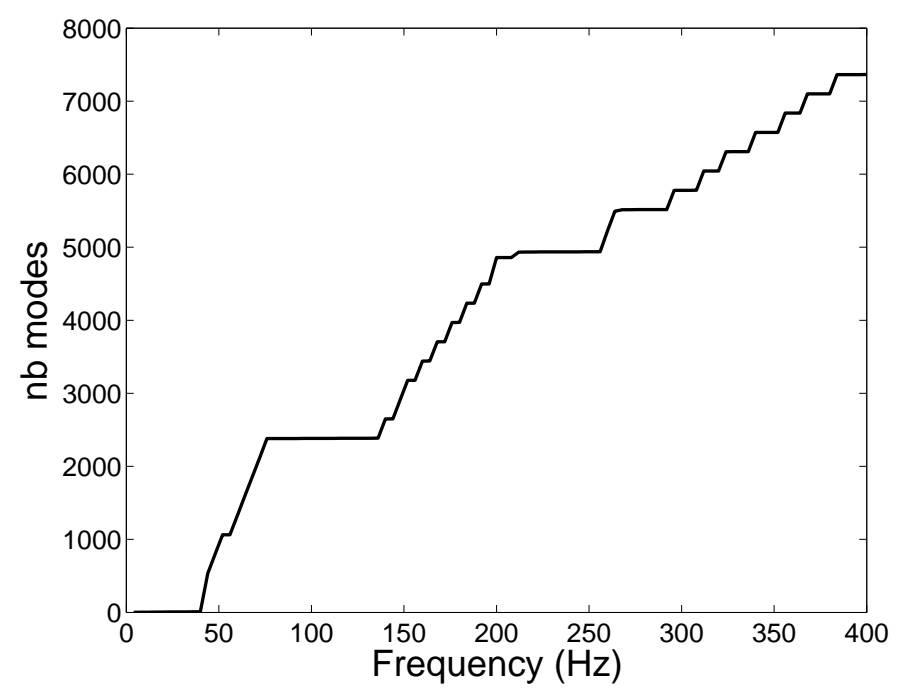

Figure 2: Cumulative modal distribution of elastic modes. 

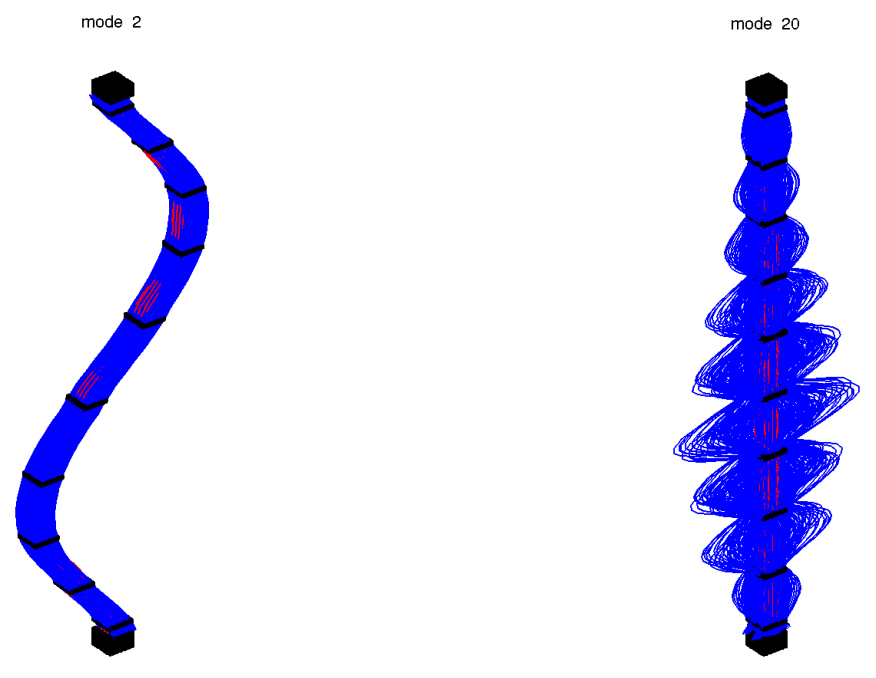

Figure 3: Left: $2^{\text {nd }}$ elastic mode (global). Right: $20^{\text {th }}$ elastic mode (local).

\section{(ii)-Row of assemblies}

Concerning the linear part, the row of assemblies is made up of seven fuel assemblies. The fuel assemblies are linked each to the others by the rigid containment building on which an homogeneous seismic displacement is imposed. The gap between two assemblies is $2.09 \times 10^{-3} \mathrm{~m}$. The gap between the leftmost assembly and the containment building is $1.9 \times 10^{-3} \mathrm{~m}$. The gap between the rightmost assembly and the containment building is $1.9 \times 10^{-3} \mathrm{~m}$. The mesh of the finite element model is plotted in Fig. 4 . The finite element model has 313, 908 elements and 3,147,060 DOFs. There are 51,548 elastic modes in the frequency band $[0,400] \mathrm{Hz}$ (each elastic mode of a single fuel assembly is reproduced seven times). The possible contact grid/grid and grid/containment are taken into account by introducing 160 elastic stops. Each grid has a left elastic stop and a right elastic stop, the containment building has 10 elastic stops face to rightmost assembly grids and 10 elastic stops face to leftmost assembly grids. 


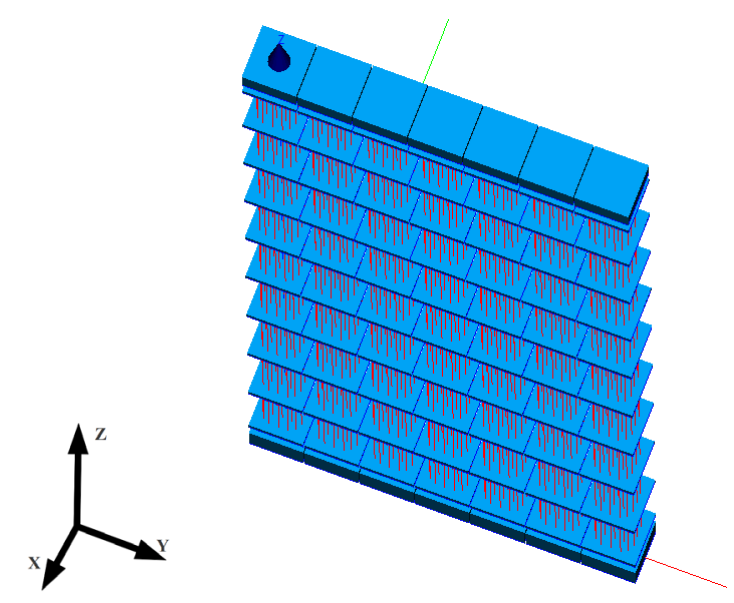

Figure 4: Finite element mesh of a row of seven fuel assemblies: Grids (blue) and stiff guide tubes (red). The flexible fuel rods are not plotted.

\subsection{Construction and validation of the reduced-order computational model}

In this section, a single fuel assembly is considered. The first step consists in the construction of the $n_{J}$ disjoint subdomains $\Omega_{j}$ introduced in Section 2.2. Since we want to filter the local transverse displacements, the subdomains are chosen as 100 slices of equal thickness. The eigenvectors $\varphi_{\alpha}^{g}$ are then computed following the method introduced in Section 2.3. In the frequency band $[0,400] \mathrm{Hz}$, there are 35 global eigenvectors. The $9^{\text {th }}$ global eigenvector is plotted in Fig. 5. In the frequency band $[0,400] \mathrm{Hz}$, the number of global eigenvectors (35) is much lower than the number of elastic modes $(7,364)$. In this paper, the local contributions for the nonlinear responses of the structure are neglected. The nonlinearities are localized at the grids which follow the global displacements of the fuel assemblies. Therefore, we have to verify that the global eigenvectors correctly span the space of global displacements. This accuracy of the reduced-order computational model could be analyzed by comparison with the reference computational model. However, the computation of the nonlinear transient responses with the reference computational model is very difficult (the presence of localized nonlinearities requires the use of a very small time step for the time integration scheme). Nevertheless, since the nonlinear global displacements space is approximated using global eigenvectors, we have only to verify the accuracy by comparing (1) the linear frequency response functions of the reduced-order 


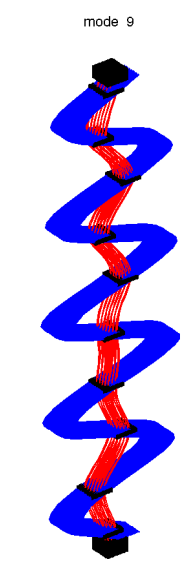

Figure 5: $9^{\text {th }}$ global eigenvector.

computational model without localized nonlinearities, with (2) the frequency response functions of the reference computational model without localized nonlinearities. The convergence analysis is then carried out for the calculation of the nonlinear transient responses in order to verify that enough global eigenvectors have been taken into account in the synthesis of the nonlinear transient responses. A Rayleigh damping model is used and is constructed for the frequencies $3 \mathrm{~Hz}$ and $400 \mathrm{~Hz}$ with a damping ratio of 0.04 . A point load is applied to the node, denoted as $P_{\text {exc }}$, which is located at the middle of the $9^{\text {th }}$ grid (from bottom to top). This load is equal to $1 \mathrm{~N}$ in the frequency band $[0,400] \mathrm{Hz}$ following the $\boldsymbol{x}$-direction. The containment building is fixed. The measurement node, denoted as $P_{\mathrm{obs}}$, is located at the middle of the $4^{\text {th }}$ grid. The frequency response functions at points $P_{\mathrm{obs}}$ and $P_{\mathrm{exc}}$ are plotted in Figs. 6 to 9. These figures show a very good accuracy of the reduced-order computational model in the frequency band $[0,100] \mathrm{Hz}$. In the frequency band $[100,300] \mathrm{Hz}$, the accuracy of the reduced-order computational model is less. These small deviations are due to the local contributions in the neighborhood of the observation points, which are not taken into account when the basis of the space of global displacements is used to construct the reduced-order computational model. 


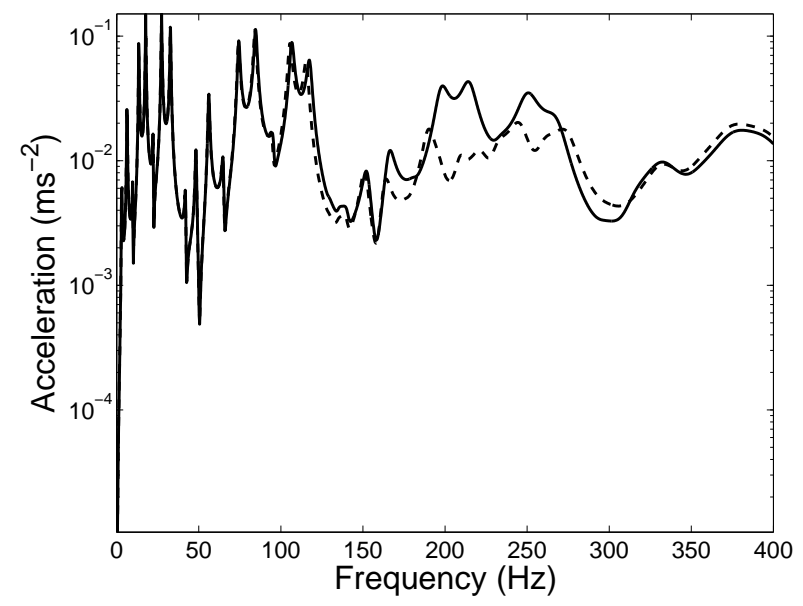

Figure 6: Modulus of the frequency response function of the acceleration in the $\boldsymbol{x}$-direction at point $P_{\mathrm{obs}}$ : reduced-order computational model (solid line) and reference computational model (dashed line).

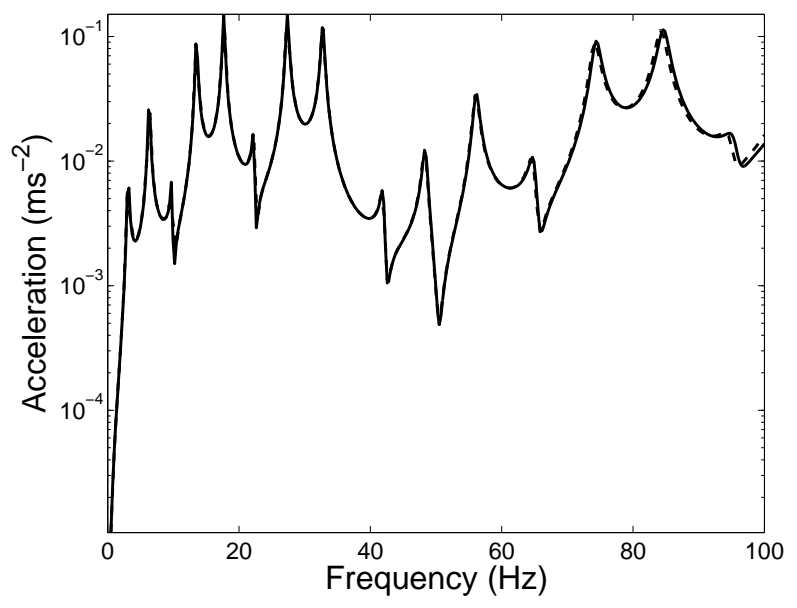

Figure 7: Modulus of the frequency response function of the acceleration in the $\boldsymbol{x}$-direction at point $P_{\text {obs }}($ zoom $[0,100] H z)$ : reduced-order computational model (solid line) and reference computational model (dashed line).

\subsection{Nonlinear transient response of a row of seven fuel assemblies}

Each fuel assembly of the row is decomposed into 100 slices yielding 700 subdomains. For the frequency band $[0,400] \mathrm{Hz}$, the reduced-order com- 


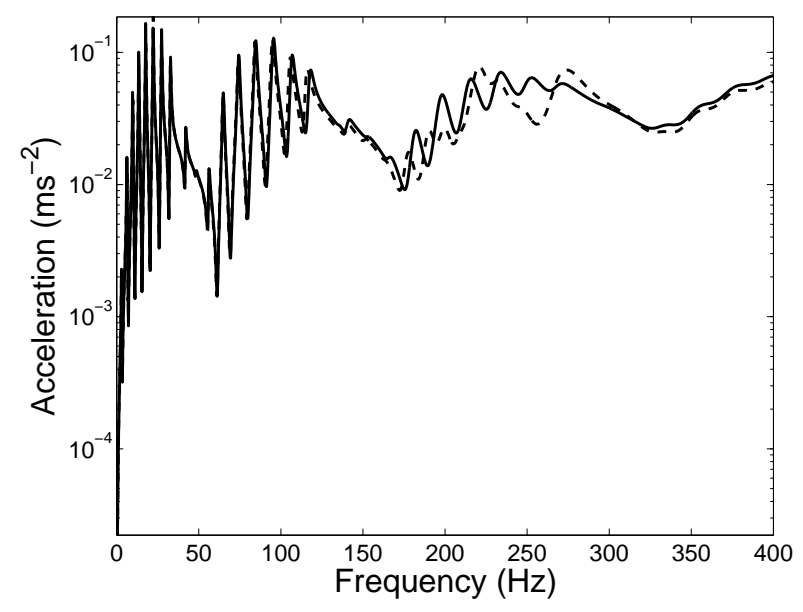

Figure 8: Modulus of the frequency response function of the acceleration in the $\boldsymbol{x}$-direction at point $P_{\text {exc }}$ : reduced-order computational model (solid line) and reference computational model (dashed line).

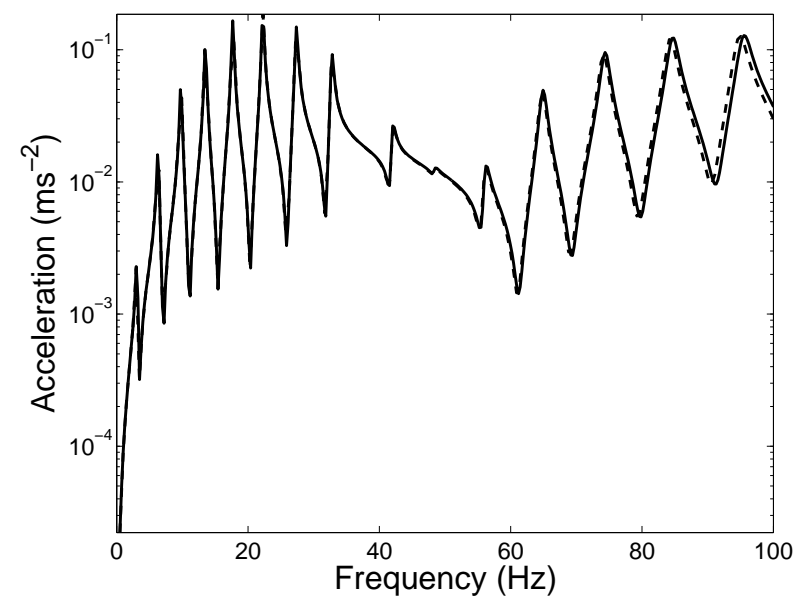

Figure 9: Modulus of the frequency response function of the acceleration in the $\boldsymbol{x}$-direction at point $P_{\text {exc }}$ (zoom $\left.[0,100] H z\right)$ : reduced-order computational model (solid line) and reference computational model (dashed line).

putational model is constructed with 245 global eigenvectors (for which the convergence is reached, see the convergence analysis below), instead of 51, 548 elastic modes which would be required with a classical modal analysis. The 
cumulative distribution of the global eigenvalues is plotted in Fig. 10. The

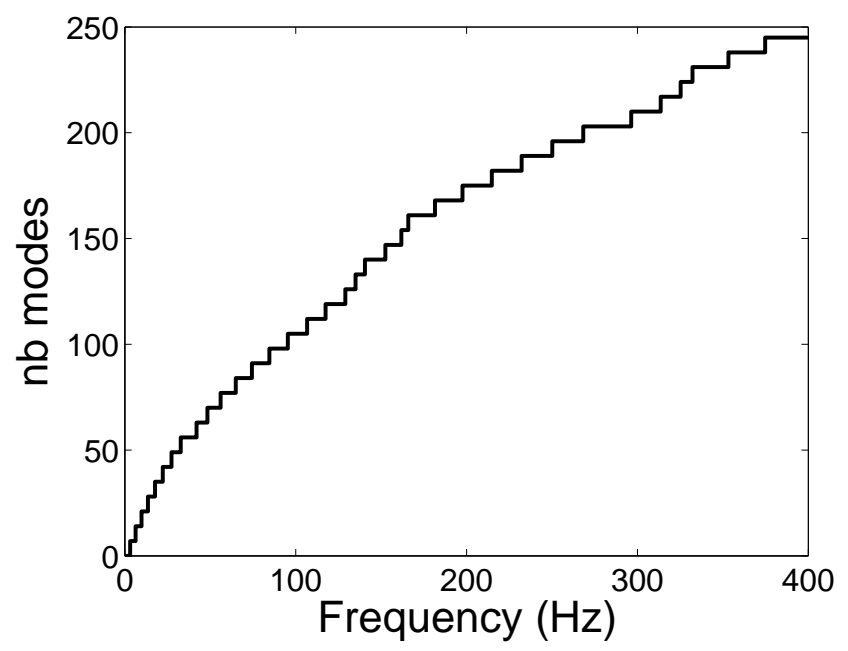

Figure 10: Cumulative distribution for the global eigenvalues for a row of seven fuel assemblies.

displacement of the containment building following the $\boldsymbol{x}$-direction is imposed and is denoted by $x_{s}(t)$. We are interested in the nonlinear transient relative displacement of the row of the seven fuel assemblies. The damping ratio is over 0.3 for the first seven global eigenmodes and the damping ratio is around 0.1 for the other global eigenmodes. The relative displacement vector is solution of Eqs. (14) to (16) with $\boldsymbol{f}(t)=-\left[\Phi^{g}\right]^{T}[\mathbb{M}] \boldsymbol{w} \ddot{x}_{s}(t)$ in which $\boldsymbol{w}$ is a vector whose components are equal to 1 for all the DOFs corresponding to the displacements following the $\boldsymbol{x}$-direction and are equal to zero for the other DOFs. The function $t \mapsto \ddot{x}_{s}(t)$ and its Fourier transform are plotted in Figs. 11 and 12. In Fig. 12, it can be seen that the energy of the imposed displacement is concentrated in the frequency band $[0,40] \mathrm{Hz}$. The nonlinear relative response is calculated in the time interval $[0,19.5] \mathrm{s}$ using an explicit Euler integration scheme with an integration time step of $10^{-5} \mathrm{~s}$. The observation point $P_{1}$ belongs to the $1^{\text {st }}$ assembly (from left to right), the observation point $P_{2}$ belongs to the $5^{\text {th }}$ assembly and the observation point $P_{3}$ belongs to the $7^{\text {th }}$ assembly. These three observation points are located at the middle of $6^{\text {th }}$ grid (from bottom to top). For points $P_{1}, P_{2}$ and $P_{3}$, the relative transient displacements are plotted in Figs. 13 to 15. The convergence of the contact forces between grids (nonlinear forces) with respect 


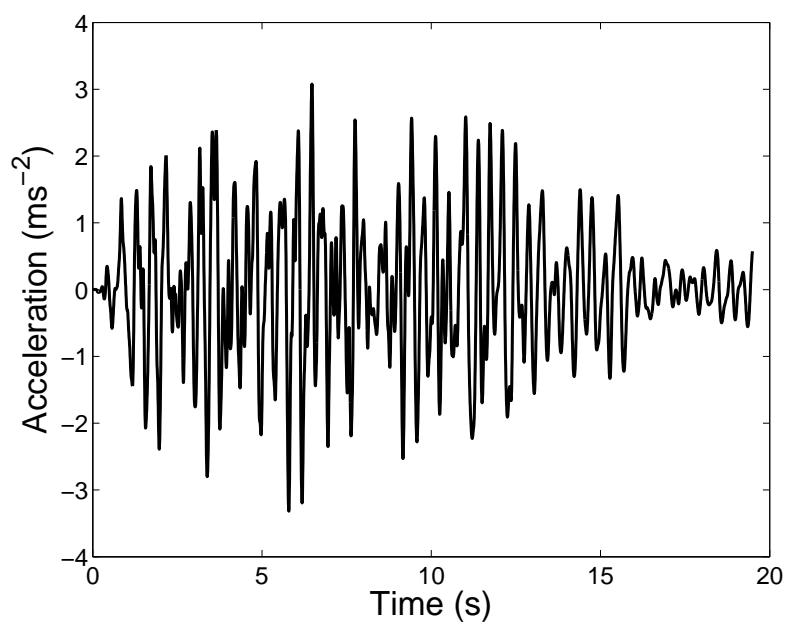

Figure 11: Function $t \mapsto \ddot{x}_{s}(t)$.

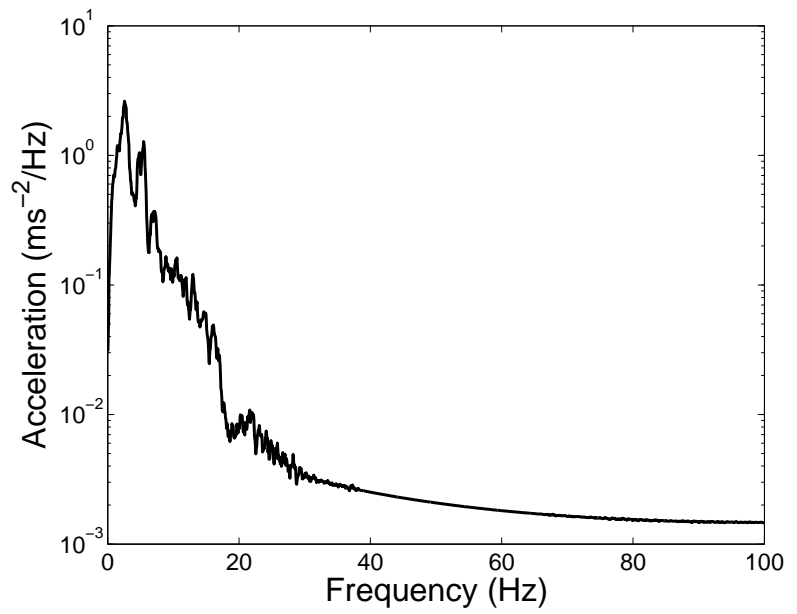

Figure 12: Modulus of the Fourier transform of function $t \mapsto \ddot{x}_{s}(t)$.

to the size of the global basis are analyzed for each elastic stop through the function $n_{g} \mapsto \int_{0}^{T} F_{N}\left(t ; n_{g}\right)^{2} d t$ in which $F_{N}\left(t, n_{g}\right)$ is the transient normal force calculated using $n_{g}$ global eigenvectors. The convergence function for points $P 1, P 2$ and $P 3$ are plotted on Figs. 16 to 18. From these figures and from Fig. 10, it can be seen that, although the energy of the imposed displacement is concentrated in the frequency band $[0,40] \mathrm{Hz}$, one need to 


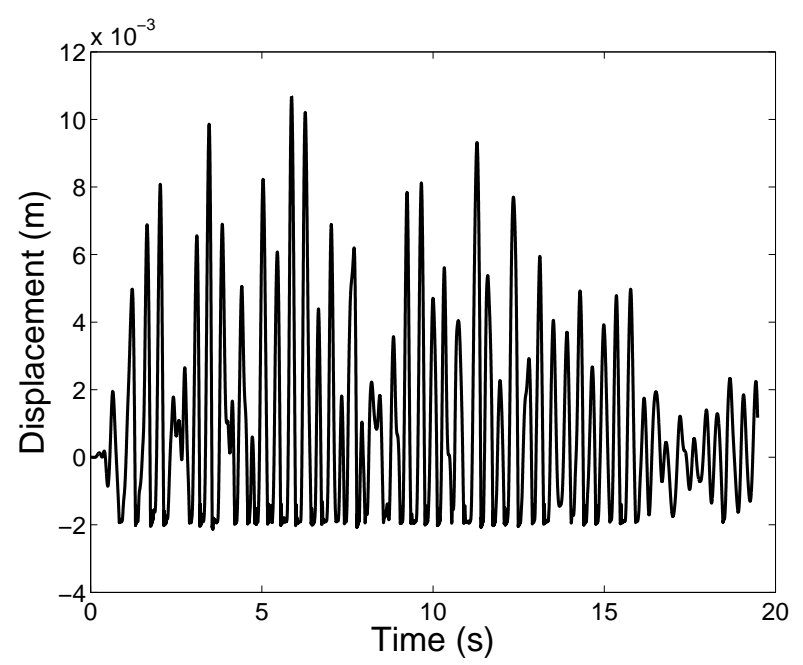

Figure 13: Relative transient displacement following the $\boldsymbol{x}$-direction for observation point $P_{1}$.

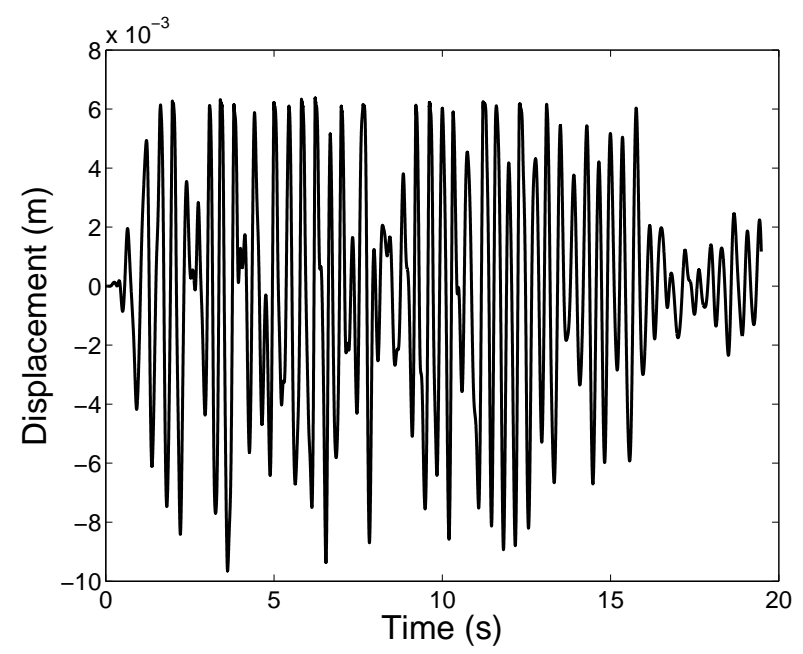

Figure 14: Relative transient displacement following the $\boldsymbol{x}$-direction for observation point $P_{2}$.

take into account higher global eigenfrequencies in order to obtain a good convergence of the nonlinear response (this would not be the case for a linear system). 


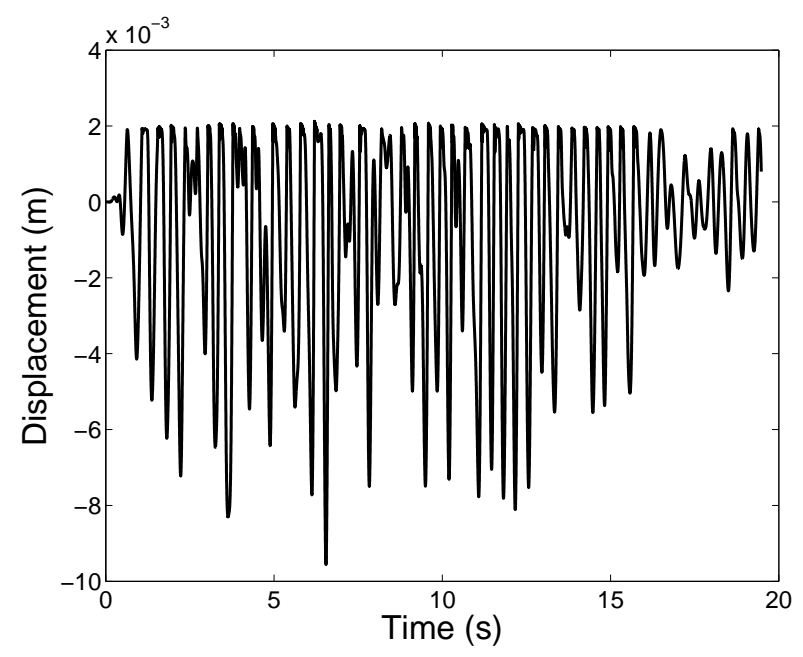

Figure 15: Relative transient displacement following the $\boldsymbol{x}$-direction for observation point $P_{3}$.

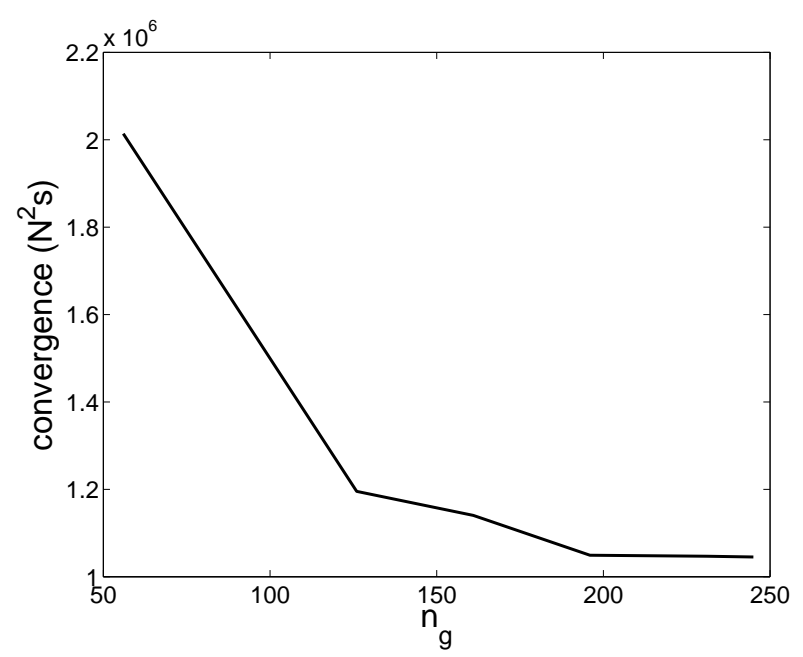

Figure 16: Convergence of the contact force for observation point $P_{1}$.

\section{Conclusions}

A method has been presented for constructing a reduced-order computational model for nonlinear dynamical structures for which there are many 


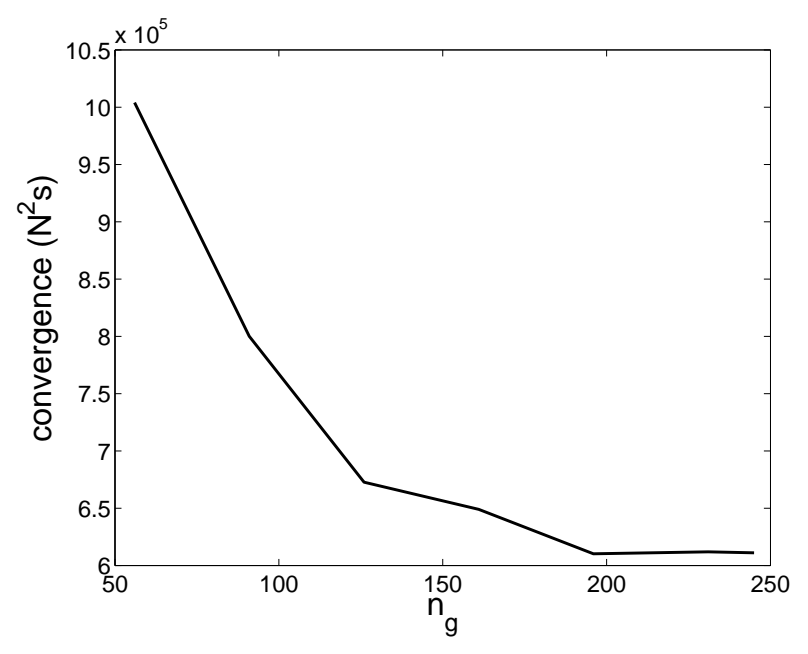

Figure 17: Convergence of the contact force for observation point $P_{2}$.

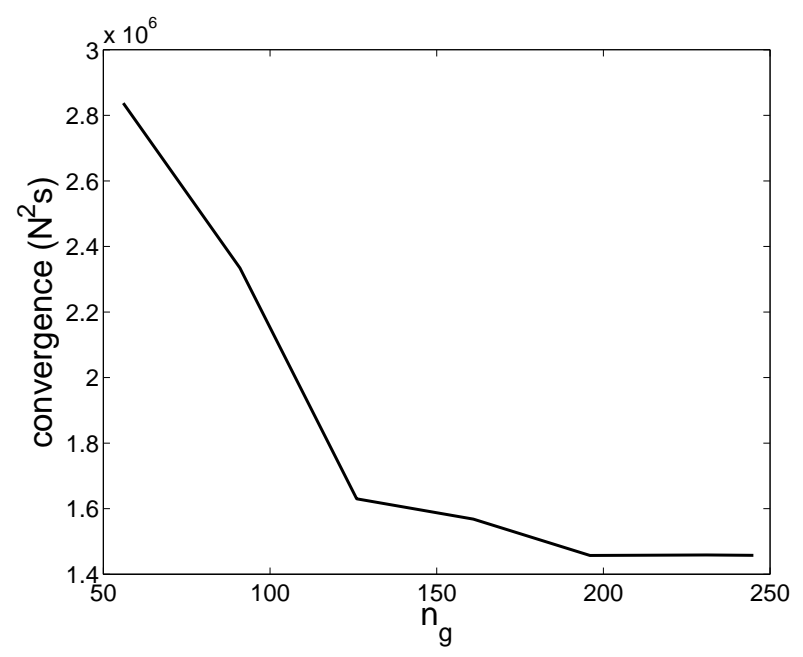

Figure 18: Convergence of the contact force for observation point $P_{3}$.

local elastic modes in the low-frequency range. This method is based on the use of a basis of the space of global displacements. The constructed reducedorder computational model has a small size and allows the displacement of the rigid part of the structure to be predicted with a good accuracy. The methodology has successfully been applied to a complex industrial dynamical 
system for which the computational model has several millions of degrees of freedom and numerous localized nonlinearities. The results show that the nonlinear dynamical response can be calculated with a good accuracy and a good convergence using only 245 global eigenvectors instead of 51, 548 elastic modes if a classical modal analysis had been used.

\section{Appendix A. Construction of matrices $\left[\boldsymbol{H}^{r}\right],\left[\boldsymbol{H}^{c}\right],\left[\mathbb{M}^{r}\right]$ and $\left[\mathbb{M}^{c}\right]$}

In this appendix, the explicit construction of matrices $\left[H^{r}\right],\left[H^{c}\right],\left[\mathbb{M}^{r}\right]$ and $\left[\mathbb{M}^{c}\right]$ are given. For such a construction, it is assumed that the sparse mass matrix $[\mathbb{M}]$ is diagonal. If it is not the case, then mass matrix $[\mathbb{M}]$ is lumped using any lumping method. The discretization of projection operator $h^{r}$ defined by Eq. (3) yields $\mathbb{U}^{r}=\left[H^{r}\right] \mathbb{U}$.

For the translation DOFs, the entries of the $(m \times m)$ real matrix $\left[H^{r}\right]$ are such that,

$$
\left[H^{r}\right]_{i j}=\frac{1}{m_{\text {ind }(i)}}[\mathbb{M}]_{j j} \delta_{i n d(i), \operatorname{ind}(j)} \delta_{\operatorname{dir}(i), \operatorname{dir}(j)} \quad,
$$

in which $\delta_{\alpha, \beta}$ is the Kronecker symbol. For all $k$ in $\left\{1, \ldots, n_{J}\right\}$, if the node relative to the DOF $\mathbb{U}_{i}$ belongs to subdomain $\Omega_{k}$, then $\operatorname{ind}(i)=k$ and, $\operatorname{ind}(i)=0$ otherwise. In Eq. (A.1), $\operatorname{dir}(i)$ is the translation direction of the DOF $\mathbb{U}_{i}(\operatorname{dir}(i)$ is equal to 1,2 or 3$)$ and $m_{k}$ is the mass of subdomain $\Omega_{k}$ such that $m_{k}=\sum_{i=1}^{m}[\mathbb{M}]_{i i} \delta_{i n d(i), k} \delta_{\operatorname{dir}(i), 1}$ (the mass is identical for the three directions). For instance, let us consider the following simple case. There are two subdomains $\Omega_{1}$ and $\Omega_{2}$ such that $\Omega=\Omega_{1} \cup \Omega_{2}$ and there is only one translation direction. The nodes of the DOFs $\mathbb{U}_{1}$ and $\mathbb{U}_{2}$ belong to subdomain $\Omega_{1}$ and the nodes of the DOFs $\mathbb{U}_{3}$ and $\mathbb{U}_{4}$ belong to subdomain $\Omega_{2}$. The diagonal mass matrix is written as $[\mathbb{M}]=\operatorname{diag}\left\{\left(m a_{1}, m a_{2}, m a_{3}, m a_{4}\right)\right\}$. Therefore, matrix $\left[H^{r}\right]$ is written as

$$
\left[H^{r}\right]=\left[\begin{array}{cccc}
m a_{1} / m_{1} & m a_{2} / m_{1} & 0 & 0 \\
m a_{1} / m_{1} & m a_{2} / m_{1} & 0 & 0 \\
0 & 0 & m a_{3} / m_{2} & m a_{4} / m_{2} \\
0 & 0 & m a_{3} / m_{2} & m a_{4} / m_{2}
\end{array}\right]
$$

in which $m_{1}=m a_{1}+m a_{2}$ and $m_{2}=m a_{3}+m a_{4}$.

For the rotation DOFs, the entries of $\left[H^{r}\right]$ are defined as follows. If $\mathbb{U}_{i}$ or 
$\mathbb{U}_{j}$ is a rotation DOF, then $\left[H^{r}\right]_{i j}=0$.

The complementary matrix $\left[H^{c}\right]$ relative to the discretization of the projection operator $h^{c}$ is then defined by $\left[H^{c}\right]=\left[I_{m}\right]-\left[H^{r}\right]$ in which $\left[I_{m}\right]$ is the $(m \times m)$ identity matrix. By analyzing the lines of matrix $\left[H^{r}\right]$, it can easily be shown that the rank of this matrix is $3 n_{J}$. Therefore the rank of matrix $\left[H^{c}\right]$ is $m-3 n_{J}$. Consequently, matrices $\left[\mathbb{M}^{r}\right]$ and $\left[\mathbb{M}^{c}\right]$ are constructed using Eqs. (7) and (8). Since mass matrix $[\mathbb{M}]$ is positive definite, it can be deduced that the rank of matrix $\left[\mathbb{M}^{r}\right]$ is $3 n_{J}$ and the rank of matrix $\left[\mathbb{M}^{c}\right]$ is $m-3 n_{J}$.

\section{Appendix B. Subspace iteration algorithm for the construction of the basis of the space of global displacements}

In this appendix, the subspace iteration algorithm [2], which is used to solve the generalized eigenvalue problem defined by Eq. (11), is summarized. The matrix $\left[\mathbb{M}^{r}\right]$ is full and therefore, cannot be assembled and stored during the calculus of the basis of the space of global displacements. The proposed method is based on the classical subspace iteration algorithm. In this appendix, the exponent $g$ is omitted in order to simplify notation.

Initialization: A random $(m \times p)$ matrix $\left[\Phi^{0}\right]$ is generated with $p \geq 2 n_{g}+8$, in which $n_{g}$ is the number of the global eigenvectors we want to extract.

Loop $i$ :

(1) Normalization of the column of $\left[\Phi^{i}\right] \rightarrow\left[\widehat{\Phi}^{i}\right]$.

(2) Calculation of $\left[\Phi_{a}^{i}\right]=[\mathbb{K}]^{-1}\left[\mathbb{M}^{r}\right]\left[\widehat{\Phi}^{i}\right]$.

Using Eq. (10), we then have $\left[\Phi_{a}^{i}\right]=[\mathbb{K}]^{-1}[\mathbb{M}]\left[H^{r}\right]\left[\widehat{\Phi}^{i}\right]=[\mathbb{K}]^{-1}[\mathbb{M}]\left[\widehat{\Phi}^{i, r}\right]$.

(3) Calculation of $\left[\tilde{K}^{i}\right]=\left[\Phi_{a}^{i}\right]^{T}[\mathbb{K}]\left[\Phi_{a}^{i}\right]$ and $\left[\tilde{M}^{r, i}\right]=\left[\Phi_{a}^{i}\right]^{T}\left[\mathbb{M}^{r}\right]\left[\Phi_{a}^{i}\right]$. We then have $\left[\tilde{M}^{r, i}\right]=\left[\Phi_{a}^{i}\right]^{T}\left[H^{r}\right]^{T}[\mathbb{M}]\left[H^{r}\right]\left[\Phi_{a}^{i}\right]=\left[\Phi_{a}^{r, i}\right]^{T}[\mathbb{M}]\left[\Phi_{a}^{r, i}\right]$.

(4) Resolution of the generalized eigenvalue problem defined by $\left(\left[\widetilde{K}^{i}\right]-\right.$ $\left.\lambda\left[\tilde{M}^{r, i}\right]\right) \boldsymbol{\varphi}_{b}=\mathbf{0} \rightarrow\left[\Phi_{b}^{i}\right]$

(5) Calculation of $\left[\Phi^{i+1}\right]=\left[\Phi_{a}^{i}\right]\left[\Phi_{b}^{i}\right]$

The convergence of eigenvectors is generally fast and needs a few iterations of the algorithm. It should be noted that $\left[H^{r}\right]$ is a full $(m \times m)$ matrix and therefore cannot be stored. Consequently, matrices $\left[\hat{\Phi}^{i, r}\right]$ and $\left[\Phi_{a}^{r, i}\right]$ are directly calculated from $\left[\hat{\Phi}^{i}\right]$ and $\left[\Phi_{a}^{i}\right]$ by using the mass matrix $[\mathbb{M}]$. 


\section{References}

[1] M.F.A. Azeez, A.F. Vakakis, Proper orthogonal decomposition (POD) of a class of vibroimpact oscillations, Journal of Sound and Vibration 240(5) (2001) 859-889.

[2] K.J. Bathe, E.L. Wilson, Numerical Methods in Finite Element Analysis, Prentice-Hall, Englewoods Cliffs, New Jersey, 1976.

[3] T. Belytschko, W.L. Mindle, Flexural Wave-propagation Behavior of Lumped Mass Approximations, Computer and Structures 12(6) (1980) 805-812.

[4] N. Bouhaddi and R. Fillod, A method for selecting master DOF in dynamic substructuring using the Guyan condensation method, Computers and Structures, 45(5-6) (1992) 941-946.

[5] I. Bucher, S.G. Braun, Left Eigenvectors: Extraction From Measurements and Physical Interpretation, Journal of Applied Mechanics Transactions of the ASME 64(1) (1997) 97-105.

[6] H.C. Chan, C.W. Cai, Y.K. Cheung, Convergence Studies of Dynamic Analysis by Using the Finite Element Method with Lumped Mass Matrix, Journal of Sound and Vibration 165(2) (1993), 193-207.

[7] R.R. Craig, A.J. Kurdila, Fundmentals of Structural Dynamics, 2nd Edition, John Wiley and Sons, New Jersey, 2006.

[8] R.J. Guyan, Reduction of Stiffness and Mass Matrices, AIAA Journal 3 (1965) 380-388.

[9] J.L. Guyader, Modal sampling method for the vibration study of systems of high modal density, J. Acoust. Soc. Am. 88(5) (1990) 2269-2270.

[10] J.L. Guyader, Characterization and reduction of dynamical models of vibratiing systems with high modal density, Journal of Sound and Vibration 328(4-5) (2009) 488-506.

[11] Y. Hahn, N. Kikuchi, Identification of global modeshape from a few nodal eigenvectors using simple free-form deformation, Engineering with Computers 21(2) (2005) 115-128. 
[12] P. Holmes, J.L. Lumley, G. Berkooz, Turbulence, Coherent Structures, Dynamical Systems and Symmetry, Cambridge University Press, Cambridge, 1997.

[13] M.S. Jensen, High Convergence Order Finite Elements With Lumped Mass Matrix, International Journal for Numerical Methods in Engineering 39(11) (1996) 1879-1888.

[14] L. Ji, B.R. Mace, Pinnington R., A mode-based approach for the mid-frequency vibration analysis of coupled long- and short-wavelength structures. Journal of Sound and Vibration 289(1) (2006) 148-1700.

[15] K. Karhunen, Zur Spektraltheorie Stochasticher Prozesse, Ann. Acad. Sci. Fennicae 220 (1945).

[16] E. Kunisch, S. Volkwein, Galerkin proper orthogonal decomposition methods for parabolic problems, Numerische Mathematik 90(1) (2001) 117-148.

[17] R.S. Langley, P. Bremmer, A Hybrid Method for the Vibration Analysis of Complex Structural-Acoustic Systems, J. Acoust. Soc. Am. 105(3) (1999) 1657-1672.

[18] W. Li, A degree selection method of matrix condensations for eigenvalue problems, Journal of Sound and Vibration, 259(2) (2003) 409-425.

[19] M. Loève, Probability Theory, 3rd ed., Van Nostrand, New York, 1963.

[20] H.G. Matthies, A. Keese, Galerkin methods for linear and nonlinear elliptic stochastic partial differential equations, Computer Methods in Applied Mechanics and Engineering, 194 (12-16) (2005) 1295-1331.

[21] A.K. Noor, M.S. Anderson, W.H. Greene, Continuum Models for Beamand Platelike-Lattice Structures, AIAA Journal 16(12) (1978) 12191228.

[22] J.H. Ong, Improved automatic masters for eigenvalue economization, Finite Element in Analysis and Design, 3 (1987) 149-160.

[23] J. Planchard, Vibration of nuclear fuel assemblies: a simplified model, Nuclear Engineering and Design 86(3) (1995) 383-391. 
[24] R. Sampaio, C. Soize, Remarks on the efficiency of POD for model reduction in nonlinear dynamics of continuous elastic systems, International Journal for Numerical Methods in Engineering 72(1) (2007) 22-45.

[25] C. Soize, Reduced models in the medium frequency range for general dissipative structural-dynamics systems, European Journal of Mechanics A/Solids 17(4) (1998) 657-685.

[26] C. Soize, A. Batou, Stochastic reduced-order model in low-frequency dynamics in presence of numerous local elastic modes, Journal of Applied Mechanics - Transactions of the ASME 78(6) (2011) 061003. 